\title{
Unraveling the Water Degradation Mechanism of $\mathrm{CH}_{3} \mathrm{NH}_{3} \mathrm{Pbl}_{3}$
}

\author{
Chao Zheng* and Oleg Rubel* \\ Department of Materials Science and Engineering, McMaster University, Hamilton \\ E-mail: zhengc8@mcmaster.ca; rubelo@mcmaster.ca
}




\begin{abstract}
Instability of perovskite photovoltaics is still a topic which is currently under intense debate, especially the role of water environment. Unraveling the mechanism of this instability is urgent to enable practical application of perovskite solar cells. Here, $a b$ initio metadynamics is employed to investigate the initial phase of a dissolution process of $\mathrm{CH}_{3} \mathrm{NH}_{3} \mathrm{PbI}_{3}\left(\mathrm{MAPbI}_{3}\right)$ in explicit water. It is found that the initial dissolution of $\mathrm{MAPbI}_{3}$ is a complex multi-step process triggered by the departure of $\mathrm{I}^{-}$ ion from the $\mathrm{CH}_{3} \mathrm{NH}_{3}$ I-terminated surface. Reconstruction of the free energy landscape indicates a low energy barrier for water dissolution of $\mathrm{MAPbI}_{3}$. In addition, we propose a two-step thermodynamic cycle for $\mathrm{MAPbI}_{3}$ dissolution in water at a finite concentration that renders a spontaneity of the dissolution process. The low energy barrier for the initial dissolution step and the spontaneous nature of $\mathrm{MAPbI}_{3}$ dissolution in water explain why the water immediately destroys pristine $\mathrm{MAPbI}_{3}$. The dissolution thermodynamics of all-inorganic $\mathrm{CsPbI}_{3}$ perovskite is also analyzed for comparison. Hydration enthalpies and entropies of aqueous ions play an important role for the dissolution process. Our findings provide a comprehensive understanding to the current debate on water instability of $\mathrm{MAPbI}_{3}$.
\end{abstract}

\title{
Introduction
}

Evolution of hybrid halide perovskite solar cells makes a contribution to the goal of replacement of fossil fuels. High power conversion efficiency and low fabrication cost raise perovskite photovoltaics as a tough competitor against the silicon solar cells. Since the inception of halide perovskite solar cells with $3.8 \%$ power conversion efficiency in $2009,{ }^{1}$ within 10 years of development, the world record power conversion efficiency of perovskite photovoltaics has reached $24.2 \%$ according to the efficiency chart published by the National Renewable Energy Laboratory. However, the poor stability of perovskite photovoltaic absorbers still remains unsolved and hinders the solar cells entering people's daily life. 
Among the stimuli caused degradation of hybrid perovskite, water is confirmed to degrade $\mathrm{MAPbI}_{3}{ }^{.2}$ Whereas, the role of water incorporation into $\mathrm{MAPbI}_{3}$ remains a topic of debate, with conflicting results reported in the literature. It is found the moisture invasion fractures the connection of $\mathrm{C}-\mathrm{N}$ in $\mathrm{CH}_{3} \mathrm{NH}_{3}(\mathrm{MA})$ and generate ammonia and hydrogen iodide $\mathrm{e}^{314}$

$$
\mathrm{CH}_{3} \mathrm{NH}_{3} \mathrm{PbI}_{3}(\mathrm{~s}) \stackrel{\mathrm{H}_{2} \mathrm{O}}{\longrightarrow}\left(-\mathrm{CH}_{2}-\right)+\mathrm{NH}_{3}(\mathrm{~g})+\mathrm{HI}(\mathrm{g})+\mathrm{PbI}_{2}(\mathrm{~s}) \text {. }
$$

Kakekhani et al..$^{[5}$ propose a super-hydrous state of water incorporation in $\mathrm{MAPbI}_{3}$ to explain deterioration of its photovoltaic properties in a moist environment. Besides, Philippe et al. ${ }^{[6}$ and Niu et al. ${ }^{[7}$ proposed a decomposition mechanism relating to the presence of $\mathrm{CH}_{3} \mathrm{NH}_{3} \mathrm{I}$ (MAI) and $\mathrm{PbI}_{2}$,

$$
\mathrm{CH}_{3} \mathrm{NH}_{3} \mathrm{PbI}_{3}(\mathrm{~s}) \stackrel{\mathrm{H}_{2} \mathrm{O}}{\longrightarrow} \mathrm{CH}_{3} \mathrm{NH}_{3} \mathrm{I}(\mathrm{aq})+\mathrm{PbI}_{2}(\mathrm{~s})
$$

In fact, two extreme water content conditions are discussed above: atmospheric moisture and liquid water. Both of the mechanisms are important. For fresh perovskite photovoltaics, moisture degradation is obviously important for the endurance. There are plenty of publications investigating the moisture induced degradation of $\mathrm{CH}_{3} \mathrm{NH}_{3} \mathrm{PbI}_{3} \cdot{ }^{[-15}$ As photovoltaics, it has to be considered that different situations the cells will encounter, e.g. after couple of years, cracks of the photovoltaic modules are inevitable. ${ }^{[16}$ At this stage, understanding of liquid water degradation mechanism becomes important. Especially, hybrid halide perovskites contain lead element. There are less investigations about the liquid water degradation pathway. ${ }^{17}\left[19\right.$ It is still unclear about the mechanism of $\mathrm{CH}_{3} \mathrm{NH}_{3} \mathrm{PbI}_{3}$ degradation in liquid water, such as the energy barrier, decomposition details.

Key challenges in understanding the degradation mechanism of $\mathrm{MAPbI}_{3}$ are the difficulties in determining the surface chemistry of the first few atomic layers of the pristine material without any exposure to ambient atmospheric conditions, and, conversely, the difficulties in measuring this same surface chemistry in real time under exposure to realistic environments. ${ }^{4}$ Molecular dynamics (MD) make it possible to explore the degradation mech- 
anism at the atomic level. Mosconi et al. 17 , Caddeo et al. $\frac{19}{19}$ simulated the heterogeneous interface between water and $\mathrm{MAPbI}_{3}$ to study its water degradation mechanism. Mosconi et al. ${ }^{17}$ observed first MAI unit dissolved within 8.5 ps using an ab initio molecular dynamics (AIMD). Caddeo et al. $\frac{19}{19}$ observed a fast dissolution of outermost MAI-terminated layers within 10 ps using classical molecular dynamics. Whereas, the details of the initial phase of the degradation process, such as energy barriers of corresponding steps, and the preference of ions leaving the surface are still missing. We performed AIMD simulation for water and $\mathrm{MAPbI}_{3}$ interface aiming to clarify the degradation mechanism of $\mathrm{MAPbI}_{3}$ in water. Contrary to previous reports of a short degradation time, our AIMD simulation of the heterogeneous interface renders no dissolution event happening within 12 ps. This phenomenon questions the immediacy of $\mathrm{MAPbI}_{3}$ degradation in water. ${ }^{2}$ During 12 ps standard AIMD simulation, we observed both $\mathrm{I}^{-}$and $\mathrm{MA}^{+}$ions trying to detach from the $\mathrm{MAPbI}_{3}$ surface. As time passed by, the ions retrace back to the $\mathrm{Pb}-\mathrm{I}$ framework cavity. Thus, the dissolution event reported in Ref. 17 could be an artefact due to a limited simulation time of about $10 \mathrm{ps}$.

The ionic nature of $\mathrm{MAPbI}_{3} \stackrel{820}{ }$ allows to draw a parallel to the research on simulation of $\mathrm{NaCl}$ dissolution. Intriguingly, Liu et al. $\frac{21}{21}$ and Chen et al. ${ }^{22}$ encountered the same situation when dissolving $\mathrm{NaCl}$ using $\mathrm{MD}$. A single ionic dissolution is a rare event, which is unlikely to happen on the time scale of an AIMD simulation. Therefore, the dissolution needs to be "driven" artificially..$^{22}$ Metadynamics $\sqrt{23} \sqrt[25]{2}$ is a powerful algorithm that can be used both for probing the free energy landscape and for accelerating rare events in systems described by complex Hamiltonians, at the classical or at the quantum level. Since the dissolution of $\mathrm{MAPbI}_{3}$ in water is viewed as a rare event, it needs a much longer simulation time to capture the dissolution, and this finding raises a question about validity of conclusions drawn from the relatively short 10 ps simulation. 19

In the current research, we propose the water degradation mechanism employing the capability of $a b$ initio metadynamics which uses computational sands to fill the initial po- 
tential valley and to force the trapped system from the initial basin and explore the energy landscape. And it gives the possibility to describe the heterogeneous interface with a large system from the atomic level, dynamically and considering finite temperature effects. Hence, the method can accelerate and capture the dissolution process of $\mathrm{MAPbI}_{3}$ in water. Here, the free energy surface (FES) of water dissolution $\mathrm{MAPbI}_{3}$ can be reconstructed based on the historical computational sands added to the basin. The obtained FES suggests a relatively low energy barrier of the first step of the dissolution process. In addition, an analysis based on a thermodynamic cycle for dissolution $\mathrm{MAPbI}_{3}$ explains the intrinsic water instability of $\mathrm{MAPbI}_{3}$. The low dissolution energy barrier and spontaneous dissolving trend together unravel the fragile nature of $\mathrm{MAPbI}_{3}$ when encountering water.

\section{Computational method}

Electronic structure calculations have been performed in the framework of DFT ${ }^{26}$ and Perdew-Burke-Ernzerhof generalized gradient approximation 27 (GGA-PBE) for the exchangecorrelation functional. Van der Waals correction is important for halide hybrid perovskite. $28 \mid 29$

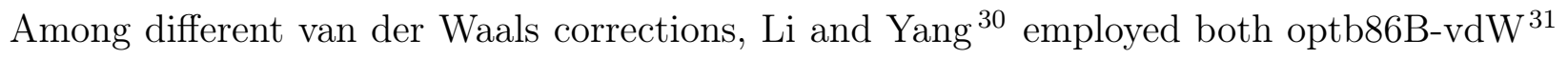
and PBE $+\mathrm{D} 3$ method $^{32}$ to optimize structures. The results show that both the vdW functionals can give accurate crystal structure predictions. And the PBE + D3 method acts even better than optb86B-vdW when comparing with experimental data. Except for the structural properties, our previous work ${ }^{29}$ analyzed the polymorphism of $\mathrm{MAPbI}_{3}$ employing different functionals including $\mathrm{PBE}+\mathrm{D} 3$. We concluded that the $\mathrm{PBE}+\mathrm{D} 3$ can accurately predict the trend of polymorphism of $\mathrm{MAPbI}_{3}$. It indicates $\mathrm{PBE}+\mathrm{D} 3$ can capture the total energy estimation very well. Hence, the PBE + D3 level van der Waals correction is considered for all calculations. Total energies of all compounds were obtained using the Vienna ab initio

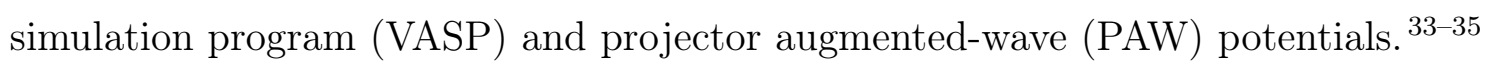

The phase separation energy difference $\Delta E_{\text {tot }}$ of $\mathrm{MAPbI}_{3}$ and $\mathrm{CsPbI}_{3}$ are adopted from our previous calculations. ${ }^{36}$ For reciprocal space integration, $3 \times 3 \times 2$ Monkhorst-Pack grid ${ }^{37}$ 
was used for tetragonal $\mathrm{MAPbI}_{3}, 4 \times 4 \times 3$ for hexagonal $\mathrm{PbI}_{2}$ and $3 \times 6 \times 2$ for orthorhombic $\mathrm{CsPbI}_{3}$. The convergence of $\Delta E_{\text {tot }}$ with respect to the k-mesh density was tested via doubling the density for investigated perovskite structures and corresponding decomposed structures. And the convergence is better than $5 \mathrm{meV}$. The cutoff energy for a plane wave expansion was set at $400 \mathrm{eV}$. The lattice constant and atomic positions were optimized such that residual forces acting on atoms did not exceed $2 \mathrm{meV} / \AA$, and the residual hydrostatic pressure was less than $50 \mathrm{MPa}$.

For AIMD calculations, a semi-empirical scaling method ${ }^{38139}$ is used to achieve a finitetemperature structure of $\mathrm{MAPbI}_{3}$ that is self-consistent with $\mathrm{PBE}$ functional with the van der Waals correction. According to scanning tunnelling electron microscopy studies of $\mathrm{MAPbI}_{3},{ }^{40141}$ we selected a MAI-terminated (001) surface structure of tetragonal MAPbI ${ }_{3}$. The $\mathrm{MAPbI}_{3}$ slab was modelled as $2 \times 2$ in plane per-optimized tetragonal supercell with the thickness of 7 atomic layers spaced by $18.6 \AA$ filled by water molecules (see Fig. 1). The number of water molecules embedded is 158 which is obtained based on the experimental liquid water density. The dimension of the periodic cell are $a=b=17.72 \AA$ which corresponds twice of the size of tetragonal $\mathrm{MAPbI}_{3}$. In total, we have $c=38.35 \AA$ for the heterogeneous structure. In order to obtain an initial randomization of the atomic positions, we performed a standard AIMD simulation in two stages: pre-heating followed by a fixed temperature relaxation. Pre-heating from 0 to $300 \mathrm{~K}$ was performed in 700 steps (step size of $1 \mathrm{fs}$ ) using a linear ramp-up function (VASP tag SMASS $=-1$ ). Velocities were scaled every $20 \mathrm{MD}$ steps. Although the orientations of $\mathrm{MA}^{+}$cations on surface are anisotropic after this two-stage relaxation, we noticed the $-\mathrm{NH}_{3}^{+}$groups of $\mathrm{MA}^{+}$ions are attracted by oxygen atoms from ajdacent water molecules during relaxation. Accuracy of computed Hellmann-Feynman forces was determined by the energy convergence criterion of $10^{-6} \mathrm{eV}$. Only one $k$ point at $\Gamma$ was used to sample the Brillouin zone. Atomic positions and velocities at the end of the preheating stage were taken as the input for the fixed temperature relaxation. The fixed temperature relaxation was conducted at $300 \mathrm{~K}$ for $\sim 9.8 \mathrm{ps}$ (step size 
of $1 \mathrm{fs}$ ). A Nosé-Hoover thermostat $\stackrel{42 / 43}{ }$ was used to stabilize the temperature (VASP tag SMASS $=0$ ). Atomic positions during AIMD were stored every 20 steps. Crystallographic information files (CIF) with atomic structures used in calculations can be accessed through the Cambridge crystallographic data centre (CCDC deposition numbers 1919295-1919299).

Metadynamics was applied to accelerates the rare events of the heterogeneous interface (VASP tag MDALGO $=21$ ). It is realized by augmenting the system Hamiltonian $\tilde{H}(t)$ with a time-dependent bias potential $\tilde{V}(t, \xi)$ which acts on selected collective variables $\xi=$ $\left\{\xi_{1}, \xi_{2}, \ldots, \xi_{m}\right\}$

$$
\tilde{H}(t)=H+\tilde{V}(t, \xi)
$$

where $H$ stands for the original Hamiltonian of unbiased system. $\tilde{V}(t, \xi)$ is defined as a sum of Gaussian hills with height $h$ and width $w$,

$$
\tilde{V}(t, \xi)=h \sum_{i=1}^{\left\lfloor t / t_{\mathrm{G}}\right\rfloor} \exp \left[-\frac{\left|\xi^{(t)}-\xi^{\left(i \cdot t_{G}\right)}\right|^{2}}{2 w^{2}}\right]
$$

During the metadynamic simulation, $\tilde{V}(t, \xi)$ is updated by adding a new Gaussian with a time increment $t_{\mathrm{G}}$ which is set to $100 \mathrm{fs}$. A collective variable $(\mathrm{CV})$ is a function of the particle positions. We employed two CVs in the current metadynamics. The first $\mathrm{CV}\left(\xi_{1}\right)$ is defined as the coordination number

$$
\xi_{1}=\sum_{i=1}^{M} \frac{1-\left(q_{i} / c_{i}\right)^{9}}{1-\left(q_{i} / c_{i}\right)^{14}}
$$

of the departing $\mathrm{I}^{-}$(or $\mathrm{N}$ atom of monitored $\mathrm{MA}^{+}$during a paralleled $\mathrm{MA}^{+}$dissolution) with the rest $\mathrm{I}^{-}$(or $\mathrm{N}$ atoms of other $\mathrm{MA}^{+}$) in the topmost complete layer of the surface. $M$ is the number of $\mathrm{I}^{-}$in the topmost layer except the monitored $\mathrm{I}^{-}\left(\right.$or $\mathrm{N}$ atom in $\mathrm{MA}^{+}$). $c_{i}$ is defined as the interatomic distance between the monitored $\mathrm{I}^{-}$(or $\mathrm{N}$ atom in $\mathrm{MA}^{+}$) and each rest of $\mathrm{I}^{-}$(or $\mathrm{N}$ atom in $\mathrm{MA}^{+}$) in the initial state. $q_{i}$ is the on-the-fly interatomic distance between the monitored $\mathrm{I}^{-}$(or $\mathrm{N}$ atom in $\mathrm{MA}^{+}$) and each rest of $\mathrm{I}^{-}$(or $\mathrm{N}$ atom in $\mathrm{MA}^{+}$) during the 
simulation.

The second $\mathrm{CV}\left(\xi_{2}\right)$ records the interatomic distance between the monitored $\mathrm{I}^{-}$and $\mathrm{Pb}^{2+}$ underneath it. An estimate of the underlying free energy $A(\xi)$ can be obtained via a sufficiently long time simulation,

$$
A(\xi)=\lim _{t \rightarrow \infty} \tilde{V}(t, \xi)+\text { const }
$$

The choice of coordination numbers and $\mathrm{Pb}-\mathrm{I}$ distance as two $\mathrm{CVs}$ is because these CVs vary according to different dissolution stages. CV $\left(\xi_{2}\right)$ directly indicates the dissolution process of the monitored $\mathrm{I}^{-}$ion. However, only $\mathrm{CV}\left(\xi_{2}\right)$ is not sufficient to tell the difference between the states of $\mathrm{I}^{-}$ion attached on surface and further dissolution in water. $\mathrm{CV}\left(\xi_{1}\right)$ is able to reflect the bond breaks accompanying the leaving of $\mathrm{I}^{-}$ion. Combination with $\mathrm{CV}\left(\xi_{1}\right)$, it is clear to explore an intermediate state. Liu et al. ${ }^{21}$ used different combination of CVs and determined that the settings same as we used are the best combination to characterize the dissolution event.

After the standard $~ 9.8$ ps MD, the width of Gaussian hill is determined from a continuous 1.8 ps metadynamics which monitors the two CVs without adding hills. The amplitudes of these CVs in the reactant well indicate the width of the well, $\underline{44}$ and we set $w=0.11$. Considering our large system (more than 800 atoms) and complexity of the dissolution procedure, we set $h=0.026 \mathrm{eV}$ from IS to IM. After passing the IM state, we increased the Gaussian height to $0.052 \mathrm{eV}$. To characterize the hydrogen bonds between water molecules and $\mathrm{I}^{-}$, we set the bonding searching range to $3.25 \AA .45$ Plumed package ${ }^{46}$ and Gnuplot were utilized to reconstruct and plot the FES of dissolution events. VESTA 3 package ${ }^{47}$ was used to visualize crystal structures. 


\section{Results and discussion}

\section{Dissolution energy barrier estimation}

In this section, it will be shown that $\mathrm{MAPbI}_{3}$ dissolution is a complex multi-step process triggered by the initial departure of $\mathrm{I}^{-}$ions from the MAI-terminated surface. The choice of the MAI-terminated surface as a starting point is based on the scanning tunnelling microscopy topography observations of halide hybrid perovskite surface. $\stackrel{40 \mid 41}{ }$ An intermediate state is identified as the departing ion is partially hydrated but still remains within a proximity from the $\mathrm{MAPbI}_{3}$ surface. Starting with an equilibrated configuration we performed metadynamics using biasing variables (see Method section) aimed at obtaining the lowest free energy pathway for the detachment of $\mathrm{I}^{-}$from the $\mathrm{MAPbI}_{3}$ surface.

We begin with the discussion of dissolving $\mathrm{I}^{-}$using the metadynamics. An equilibrated heterogeneous interface is taken as the initial structure for metadynamic simulation of the dissolution process. The observation of $\mathrm{I}^{-}$or $\mathrm{MA}^{+}$ion backtracking to $\mathrm{Pb}-\mathrm{I}$ cavity in the previous discuss is due to the trapping of the system in the initial FES basin using standard AIMD. The configuration trapped at this basin is named as the initial state (IS) shown in Fig. 1. In the IS, the monitored $\mathrm{I}^{-}$(labelled yellow) forms hydrogen bonds with two water molecules above it, and bonds with a lead atom underneath it. Under the action of the metadynamic bias, the system is discouraged to revisit previous explored spots. With accumulating the computational sands, the initial basin is filled and the system is forced to escape from the local minima. We observe the elongation of the $\mathrm{I}-\mathrm{Pb}$ bond shown in Fig 2 and Fig 2 b. After around 7 ps of metadynamic simulation, the system arrives at the first transition state $\left(\mathrm{TS}^{\mathrm{I}}\right)$ of the dissolution process shown in Fig. $2 \mathrm{c}$ and Fig. $2 \mathrm{~d}$, the $\mathrm{I}-\mathrm{Pb}$ bond breaks. At the transition state, the $\mathrm{I}^{-}$ion retains two hydrogen bonds with two water molecules. Besides, the $\mathrm{I}^{-}$forms bonds with a hydrogen atom on $-\mathrm{NH}_{3}^{+}$group of one $\mathrm{MA}^{+}$, and with a hydrogen atom on $-\mathrm{CH}_{3}$ group of the another $\mathrm{MA}^{+}$. The interatomic distance of the monitored $\mathrm{I}-\mathrm{Pb}$ bond streches from $3.22 \AA$ at IS to $5.27 \AA$ at TS . We 


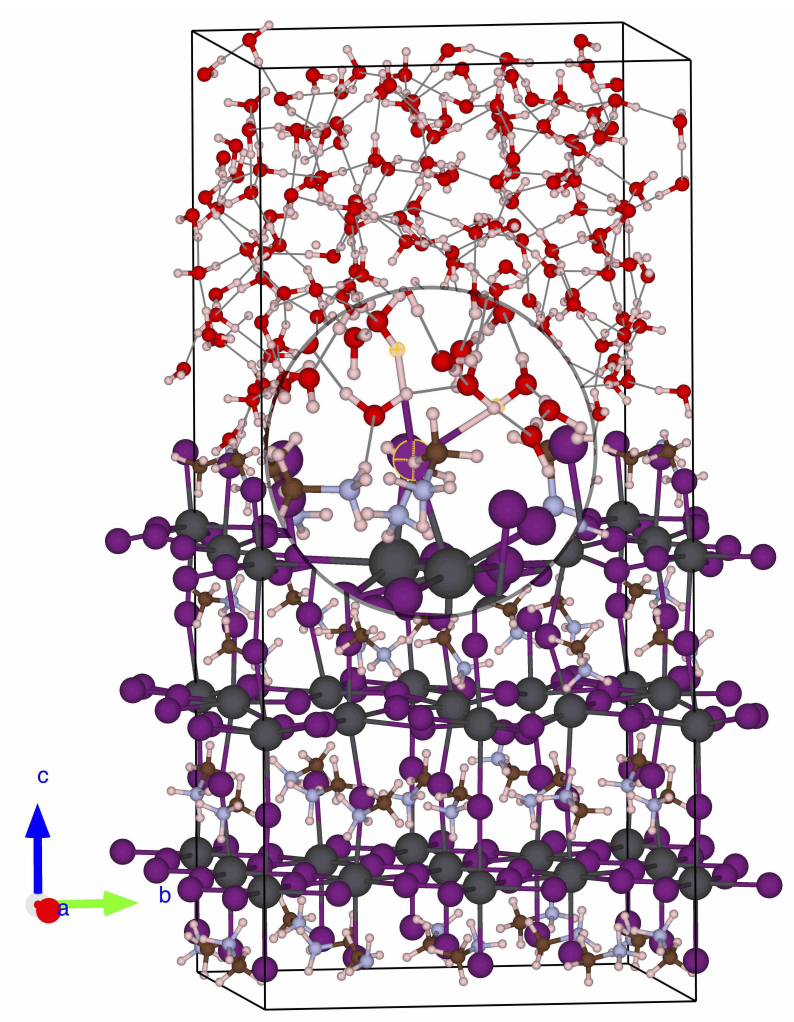

Figure 1: The initial state (IS) configuration of water $/ \mathrm{MAPbI}_{3}$ interface. Light pink represents hydrogen atoms. Red represents oxygen atoms. Brown represents carbon atoms. Light purple represents nitrogen atoms. Grey represents lead atoms. Purple represents iodine atoms.

continued the metadynamic simulation after conquering the $\mathrm{TS}^{\mathrm{I}}$. The lifted $\mathrm{I}^{-}$drifts away from the underneath $\mathrm{Pb}^{2+}$. Meanwhile, neighbour $\mathrm{MA}^{+}$cations of the monitored $\mathrm{I}^{-}$drift towards the cavity. It is intriguing to find that the departing $\mathrm{I}^{-}$ion does not enter solvent immediately and the surrounding $\mathrm{MA}^{+}$cations do not leave the lattice following the $\mathrm{I}^{-}$ion. The heterogeneous interface evolves towards a state in which the $\mathrm{I}^{-}$is partly hydrated, but remains trapped close to the surface illustrated in Fig. 2e and Fig. 2f. We assign this local energy minimum as an intermediate state (IM). The partially hydrated $\mathrm{I}^{-}$is in an adatomlike configuration. Upon transition from $\mathrm{TS}^{\mathrm{I}}$ to IM, the coordination number of the $\mathrm{I}^{-}$with the solvent water molecules increases from $\sim 2$ to $\sim 4$.

During the whole simulation, we find the system spends a long time at IM basin. The partly hydrated $\mathrm{I}^{-}$stays on top of one neighbour $\mathrm{MA}^{+}$cation due to an electrostatic attrac- 


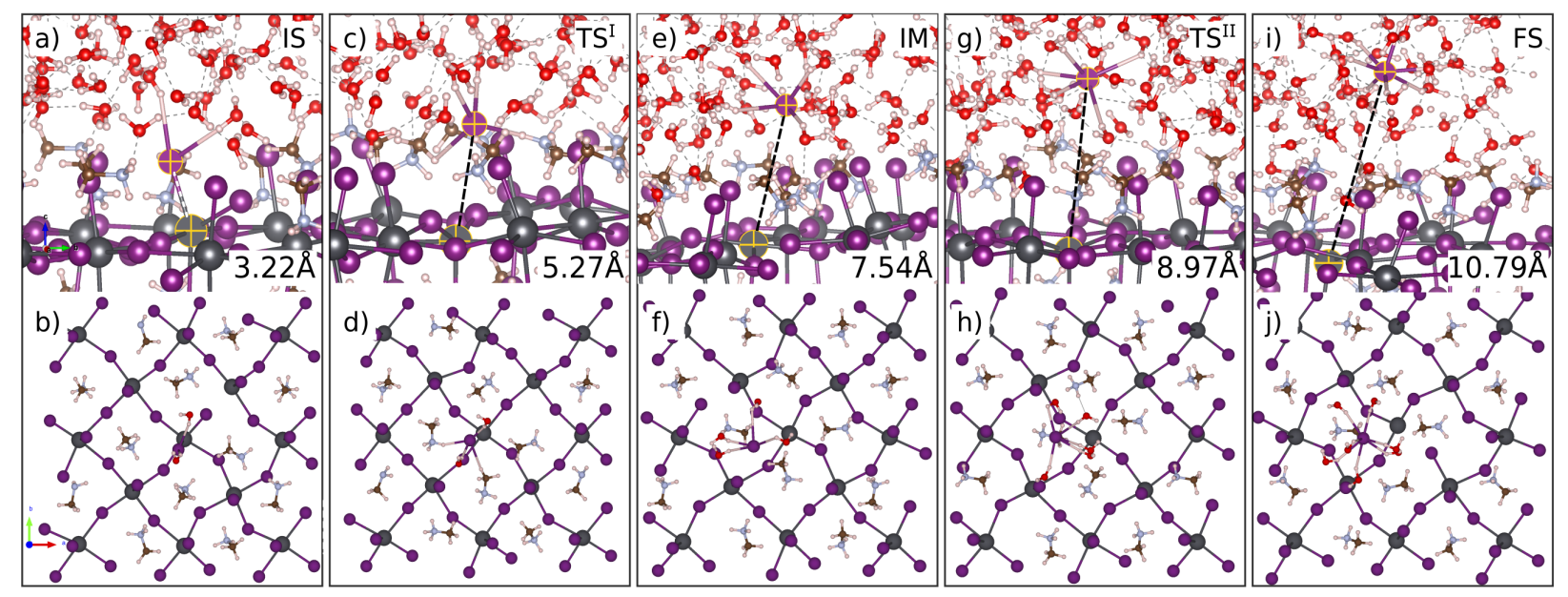

Figure 2: The initial state (IS), first transition state $\left(\mathrm{TS}^{\mathrm{I}}\right)$, intermediate state (IM), second transition state $\left(\mathrm{TS}^{\mathrm{II}}\right)$ and final state $(\mathrm{FS})$ of the initial dissolution of $\mathrm{MAPbI}_{3}$ in water, respectively. $\mathrm{I}-\mathrm{Pb}$ interaction is marked as dashed line, and the value is shown in each configuration. It is clear to see that the $\mathrm{I}-\mathrm{Pb}$ bond breaks at $\mathrm{TS}^{\mathrm{I}}$ and the monitored $\mathrm{I}^{-}$detaches surface at $\mathrm{TS}^{\mathrm{II}}$. Lower panel shows the conrresponding topview of each configuration.

tion. Once the IM basin is filled, the system comes to the second transition state $\left(\mathrm{TS}^{\mathrm{II}}\right)$, the corresponding configuration is shown in Fig. 2s and Fig. 2h. From IM to TS ${ }^{\mathrm{II}}$, the pulls from water molecules acting on the $\mathrm{I}^{-}$gradually overwhelm the interactions between the $\mathrm{I}^{-}$ and underneath $\mathrm{MA}^{+}$. The adatom-like $\mathrm{I}^{-}$ion detaches from the $\mathrm{MA}^{+}$cation. The number of water molecules in the hydration shell of $\mathrm{I}^{-}$increase to 5 . The system needs to conquer the barrier of $0.22 \mathrm{eV}$ to reach $\mathrm{TS}^{\mathrm{II}}$. This relatively larger energy barrier (compared with the initial $0.16 \mathrm{eV}$ ) is explained by breaking of the electrostatic attraction between the leaving $\mathrm{I}^{-}$and $\mathrm{MA}^{+}$. After passing through the $\mathrm{TS}^{\mathrm{II}}$, the system evolves to a final state (FS) shown in Fig. 2 and Fig. 2j. We characterize the FS as a state where $\mathrm{I}^{-}$escapes from the $\mathrm{MAPbI}_{3}$ surface and fully dissolves in water. In the FS, $\mathrm{I}^{-}$ion is coordinated by $\sim 7$ water molecules, consistent with both experimental and ab initio simulated coordination number of $6-9.45548$ The overall energy barrier for the initial dissolution event is obtained as the energy difference between the IS and $\mathrm{TS}^{\mathrm{II}}$ which is $0.18 \mathrm{eV}$.

We portray the FES of the dissolution process of $\mathrm{MAPbI}_{3}$ in water in Fig. 3. The ease of 

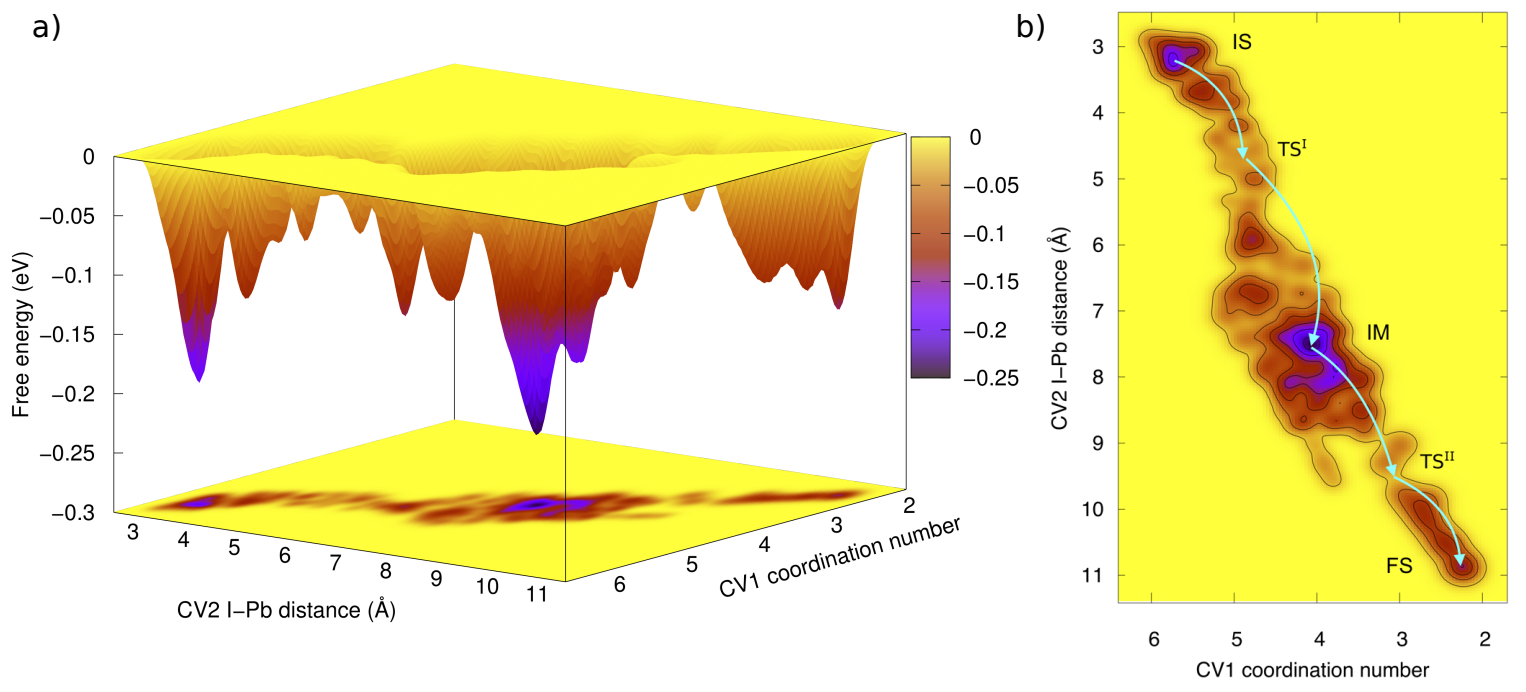

Figure 3: Reconstructed free energy surface of dissolution of $\mathrm{MAPbI}_{3}$ in water from metadynamic simulation. a) gives the free energy landscape of the dissolution process of $\mathrm{MAPbI}_{3}$ in water. b) is the contour plot of the free energy surface. Arrows point steps on the dissolution pathway.

the first dissolution event happening is determined by an energy barrier between IS and TS . Figure 3 suggests that the initial configuration needs to conquer $0.16 \mathrm{eV}$ energy barrier to break the $\mathrm{I}-\mathrm{Pb}$ bond in aqueous environment to reach TS ${ }^{\mathrm{I}}$. For comparison, Caddeo et al. 19 reported an energy barrier of $0.36 \mathrm{eV}$ consisting of a layer-by-layer degradation of $\mathrm{MAPbI}_{3}$ in water. Lin et al. $\stackrel{49}{~ r e p o r t e d ~ a ~ l a r g e r ~ e n e r g y ~ b a r r i e r ~(g r e a t e r ~ t h a n ~} 0.5 \mathrm{eV}$ ) when dissolving an $\mathrm{I}^{-}$from $\mathrm{CsPbI}_{3}$ surface in water. Releasing the $\mathrm{I}^{-}$is a competition between the hydration by water molecules and the electrostatic attraction to atoms on the surface and in the bulk of $\mathrm{MAPbI}_{3}$. From a thermodynamic point of view, the dissolution phenomenon is an effect of compensating electrostatic energy stored in the $\mathrm{MAPbI}_{3}$ surface by heat released from the hydration of the $\mathrm{I}^{-}$. This process is further promoted by the entropy gain during $\mathrm{I}^{-}$entering 
water. Detailed thermodynamic analysis of the overall dissolution process is discussed in the following section. Both the hydrophilicity of $\mathrm{I}^{-}$and $\mathrm{MA}^{+}$ion and the intrinsic low electrostatic energy of $\mathrm{MAPbI}_{3}{ }^{8 \mid 36}$ contribute to these very low energy barriers in Fig. 3.

Simulations of $\mathrm{NaCl}$ dissolution in water identify a clear preference for the dissolution of $\mathrm{Cl}^{-}$as the initial step over $\mathrm{Na}^{+} . \stackrel{2150 \mid 51}{2}$ To investigate the preference of ions leaving $\mathrm{MAPbI}_{3}$ surface at the beginning of the dissolution process, we performed an alternative metadynamic simulation where the dissolution starts with $\mathrm{MA}^{+}$. The FES of dissolution of $\mathrm{MA}^{+}$is constructed and it renders a deeper energy basin $(\sim 0.31 \mathrm{eV})$, shown in Fig. S1. However, we only obtained the FES for the inital basin of the dissolution of $\mathrm{MA}^{+}$. We find that the initial basin of $\mathrm{MA}^{+}$dissolution is much wider and deeper than the case of $\mathrm{I}^{-}$ion. Although we use relative large Gaussian height and weight, it is hard to explore the sequent stages of $\mathrm{MA}^{+}$dissolution in water. A larger Gaussian will accelerate the exploration, however, sacrifice of accuracy is expected. Due to the capability of our facility and limited time, we stopped here for exploring the sequent FES of MA+ dissolution in water. The shape and rotational property of $\mathrm{MA}^{+}$ion may contribute to the large initial basin.

The difference of the initial basins indicates $\mathrm{I}^{-}$is relatively easier to dissolve in water. Although $-\mathrm{NH}_{3}^{+}$group of $\mathrm{MA}^{+}$is hydrophilic, the connected $-\mathrm{CH}_{3}$ group is hydrophobic. When dissolving $\mathrm{MA}^{+}$in water, the hydrophobic $-\mathrm{CH}_{3}$ group needs more space to settle in water molecules. $\frac{52}{5}$ Seeking for more space in water molecule network and breaking the interactions of $\mathrm{MA}^{+}$with surrounding ions in $\mathrm{MAPbI}_{3}$ surface together lead to a higher energy barrier for the first dissolution of $\mathrm{MA}^{+}$. As a result, the lower energy barrier suggests a priority of $\mathrm{I}^{-}$leaving the surface. Note that in the supporting information of Caddeo et al. $\stackrel{19}{ }$, their simulation indicates that the first step of the dissolution MAI-terminated surface in water is releasing $\mathrm{MA}^{+}$at 5 ps. Differently, during our over 33.5 ps simulation time including equilibrium relaxation and metadynamics, we only observe the dissolution of one $\mathrm{I}^{-}$ion in water. Liu et al. $\stackrel{21}{ }$ indicated a forcefield-based description of $\mathrm{NaCl}$ dissolution in water failed to capture a preference for $\mathrm{Cl}^{-}$over $\mathrm{Na}^{+}$dissolution. Limitations related to a 
proper description of bond breaking or formation in the empirical potential framework may contribute to this discrepancy.

It is worthy to note that we didn't observe any decomposition of $\mathrm{MA}^{+}$cation during the whole simulation. Compared with the high deprotonation energy $(\sim 4.03 \mathrm{eV})$ of $\mathrm{MA}^{+}$ cation,, 53 the low energy barrier of releasing $\mathrm{I}^{-}$suggests the initial degradation $\mathrm{MAPbI}_{3}$ in water is the dissolution of MAI-terminated layer into water solute. It is meaningful to mention that the energy barrier of transformation of the $\mathrm{PbI}_{2} 2 \mathrm{D}$-planer layer to $\mathrm{PbI}_{2}$ trigonal configuration is around $0.26 \mathrm{eV} \cdot \underline{54}$ Considering the low energy barriers of dissolving $\mathrm{I}^{-}$in water as well the low energy barriers of decomposition of $\mathrm{Pb}-\mathrm{I}$ layer, the overall degradation energy barrier of $\mathrm{MAPbI}_{3}$ dissolution in water is less than $0.3 \mathrm{eV}$.

\section{Thermodynamics of $\mathrm{MAPbI}_{3}$ dissolution in water}

Followed by the discussion of the initial process of $\mathrm{MAPbI}_{3}$ dissolution in water, the thermodynamic analysis of the overall decomposition is proceeded in this section. It is also intriguing to investigate how $\mathrm{CsPbI}_{3}$ reacts with water as a comparison. The dissolution of $\mathrm{MAPbI}_{3}$ via water can be described as follow:

$$
\mathrm{CH}_{3} \mathrm{NH}_{3} \mathrm{PbI}_{3}(\mathrm{~s}) \stackrel{\Delta G_{\text {diss }}}{\longrightarrow} \mathrm{CH}_{3} \mathrm{NH}_{3}^{+}(\mathrm{aq})+\mathrm{I}^{-}(\mathrm{aq})+\mathrm{PbI}_{2}(\mathrm{~s}) \text {. }
$$

Estimation of the change of the Gibbs free energy $\left(\Delta G_{T}^{\circ}\right)$ between the reactants and products is a standard approach for predicting whether a reaction or process will occur spontaneously. Combination of the density functional theory (DFT) calculation with additional thermodynamic data (see Chap. 7 in Ref. 55 ) is used to predict $\Delta G_{T}^{\circ}$ of phase separation of $\mathrm{MAPbI}_{3} \cdot{ }^{56}$ Kye et al. ${ }^{57}$ utilize an $a b$ initio thermodynamic formalism with the effect of solution to investigate the behaviour of defects on phase stability of $\mathrm{CsPbI}_{3}$. Here, we apply the combination of theoretical and experimental data to estimate the Gibbs free energy change $\left(\Delta G_{\text {diss }}\right)$ of $\mathrm{MAPbI}_{3}$ dissolution in water at temperature $T$ as stated in Eq. (7). According 

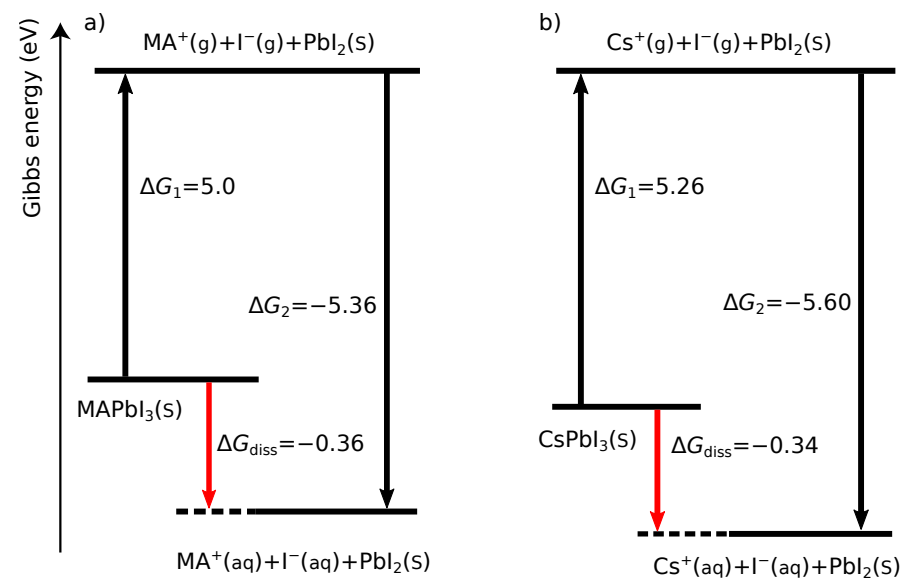

Figure 4: A thermodynamic cycle for the calculation of the dissolution Gibbs free energy change $\left(\Delta G_{\text {diss }}\right)$ for (a) $\mathrm{MAPbI}_{3}$ and (b) $\mathrm{CsPbI}_{3}$ in water.

to Sholl and Steckel ${ }^{[55}, G_{T}^{\circ}$ can be expressed as,

$$
G_{T}^{\circ}=E_{\mathrm{tot}}+\tilde{\mu}_{T}^{\circ} .
$$

Here, $E_{\text {tot }}$ is the standard state enthalpy at zero temperature which is evaluated based on DFT total energy calculations. $\tilde{\mu}_{T}^{\circ}$ captures finite temperature effects on the chemical potentials of species involved which is evaluated from NIST-JANAF thermochemical tables as well as other experimental resources.

In order to capture $\Delta G_{\text {diss }}$ at finite temperature, we designed a two-step thermodynamic cycle as shown in Fig. 4a. The first step of the thermodynamic cycle is the dissociation of $\mathrm{MAPbI}_{3}(\mathrm{~s})$ to oppositely charged ions $\mathrm{MA}^{+}(\mathrm{g})$ and $\mathrm{I}^{-}(\mathrm{g})$ and $\mathrm{PbI}_{2}(\mathrm{~s})$,

$$
\mathrm{CH}_{3} \mathrm{NH}_{3} \mathrm{PbI}_{3}(\mathrm{~s}) \stackrel{\Delta G_{1}}{\longrightarrow} \mathrm{CH}_{3} \mathrm{NH}_{3}^{+}(\mathrm{g})+\mathrm{I}^{-}(\mathrm{g})+\mathrm{PbI}_{2}(\mathrm{~s}) \text {. }
$$

During this process, finite temperature effect on enthalpies and entropies of the system from solid inital state to intermediate gaseous state and $\mathrm{PbI}_{2}(\mathrm{~s})$ are evaluated, repectively

$$
\tilde{\mu}_{T}^{\circ}=H_{T}^{\circ}-H_{0 \mathrm{~K}}^{\circ}-T S_{T}^{\circ} .
$$


The addition of the resultant $\tilde{\mu}_{T}^{\circ}$ and calculated $E_{\text {tot }}$ generates $G_{T}^{\circ}$ of each species. Related thermodynamic properties of species involved in the first step of the thermodynamic cycle of $\mathrm{MAPbI}_{3}$ dissolution in water are listed in Table 1. According to Table 1, it is feasible to Table 1: Electronic total energy $E_{\text {tot }}$ per formula unit (f.u.), $H_{T}-H_{0 \mathrm{~K}}$, standard state entropy $S_{T}^{\circ}$, the chemical potential $\tilde{\mu}_{T}^{\circ}$ and standard Gibbs free energy $G_{T}^{\circ}$ in the first step of the thermodynamic cycle involving $\mathrm{MAPbI}_{3}$ dissolution in water. Here, we focus on room temperature $T=298.15 \mathrm{~K}$.

\begin{tabular}{rccccc}
\hline Species & $\begin{array}{c}E_{\text {tot }} \\
(\mathrm{eV} / \text { f.u. })\end{array}$ & $\begin{array}{c}H_{T}-H_{0 \mathrm{~K}} \\
(\mathrm{~kJ} / \mathrm{mol})\end{array}$ & $\begin{array}{c}S_{T}^{\circ} \\
\left(\mathrm{J} \mathrm{mol} \mathrm{K}^{-1} \mathrm{~K}^{-1}\right)\end{array}$ & $\begin{array}{c}\tilde{\mu}_{T}^{\circ} \\
(\mathrm{eV} / \mathrm{f} . \mathrm{u} .)\end{array}$ & $\begin{array}{c}G_{T}^{\circ} \\
(\mathrm{eV} / \mathrm{f} . \mathrm{u} .)\end{array}$ \\
\hline $\mathrm{MAPbI}_{3}(\mathrm{~s})$ & -50.93 & $44.79^{a}$ & $374.13^{a}$ & -0.69 & -51.62 \\
$\mathrm{PbI}_{2}(\mathrm{~s})$ & -8.63 & $19.50^{b}$ & $174.84^{b}$ & -0.34 & -8.97 \\
$\mathrm{MA}^{+}(\mathrm{g})$ & -32.84 & $6.20^{b}$ & $327.7^{b}$ & -0.95 & -33.79 \\
$\mathrm{I}^{-}(\mathrm{g})$ & -3.40 & $6.20^{b}$ & $169.26^{b}$ & -0.46 & -3.86 \\
\hline $\mathrm{CsPbI}_{3}(\mathrm{~s})$ & -14.25 & $20.28^{c}$ & $219.61^{c}$ & $-0.47^{c}$ & -14.72 \\
$\mathrm{Cs}^{+}(\mathrm{g})$ & 3.83 & $6.20^{b}$ & $169.84^{b}$ & -0.46 & 3.37 \\
\hline $\mathrm{MAI}(\mathrm{s})$ & -42.36 & $22.25^{d}$ & $159.7^{d}$ & -0.26 & -5.69 \\
$\mathrm{CsI}(\mathrm{s})$ & -5.45 & $13.50^{e}$ & $123.1^{e}$ & -0.24 & -42.62
\end{tabular}

${ }^{a}$ Data obtained from Ref. $58 ;{ }^{b}$ Data extracted from the NIST-JANAF thermochemical tables; ${ }^{c}$ Data extracted from Refs. 59,60; ${ }^{d}$ Data obtained from Ref. 61, ${ }^{e}$ Data obtained from Ref. 62,

calculate the change in Gibbs free energy $\Delta G_{1}$ for the first step

$$
\Delta G_{1}=G_{T, \mathrm{MA}^{+}(\mathrm{g})}^{\circ}+G_{T, \mathrm{I}^{-}(\mathrm{g})}^{\circ}+G_{T, \mathrm{PbI}_{2}}^{\circ}-G_{T, \mathrm{MAPbI}_{3}}^{\circ} .
$$

The calculated $\Delta G_{1}=5.0 \mathrm{eV}$ is shown in Fig. 4a. The second step of the designed cycle of $\mathrm{MAPbI}_{3}$ dissolution in water involves the hydration of $\mathrm{MA}^{+}(\mathrm{g})$ and $\mathrm{I}^{-}(\mathrm{g})$

$$
\mathrm{MA}^{+}(\mathrm{g})+\mathrm{I}^{-}(\mathrm{g})+\mathrm{PbI}_{2}(\mathrm{~s}) \stackrel{\Delta G_{2}}{\longrightarrow} \mathrm{MA}^{+}(\mathrm{aq})+\mathrm{I}^{-}(\mathrm{aq})+\mathrm{PbI}_{2}(\mathrm{~s})
$$

The chemical potentials of aqueous ions $\tilde{\mu}_{T}^{\circ}(\mathrm{aq})$ in this step can be obtained via

$$
\tilde{\mu}_{T}^{\circ}(\mathrm{aq})=\tilde{\mu}_{T}^{\circ}+\Delta H_{\mathrm{hyd}}-T\left(S_{T}^{\circ}(\mathrm{aq})-S_{T}^{\circ}\right) .
$$

$S_{T}^{\circ}(\mathrm{aq})$ is the entropy of an aqueous ion. Decreased enthalpies and entropies due to hydra- 
tion process contribute to the values of $G_{T, \mathrm{MA}^{+}(\mathrm{aq})}^{\circ}$ and $G_{T, \mathrm{I}^{-}(\mathrm{aq})}^{\circ}$. Related thermodyanmic properties of the second step are shown in Table 2 .

Table 2: Hydration enthalpy $\Delta H_{\text {hyd }}^{\circ}$, entropy $S_{T}^{\circ}$ (aq) of aqueous ions, and Gibbs free energy $G_{T}^{\circ}$ of species involved in the second step of the thermodynamic cycle of $\mathrm{MAPbI}_{3}$ dissolution in water at room temperature $298.15 \mathrm{~K}$.

\begin{tabular}{rcccc}
\hline Species & $\begin{array}{c}\Delta H_{\text {hyd }}^{\circ} \\
(\mathrm{kJ} / \mathrm{mol})\end{array}$ & $\begin{array}{c}S_{T}^{\circ}(\mathrm{aq}) \\
\left(\mathrm{J} \mathrm{mol}{ }^{-1} \mathrm{~K}^{-1}\right)\end{array}$ & $\begin{array}{c}\tilde{\mu}_{T}^{\circ}(\mathrm{aq}) \\
(\mathrm{eV} / \mathrm{f} . \mathrm{u} .)\end{array}$ & $\begin{array}{c}G_{T}^{\circ} \\
(\mathrm{eV} / \mathrm{f} . \mathrm{u} .)\end{array}$ \\
\hline $\mathrm{MA}^{+}(\mathrm{aq})$ & $-284.6^{a}$ & $142.7^{b}$ & -3.33 & -36.17 \\
$\mathrm{I}^{-}(\mathrm{aq})$ & $-305.0^{c}$ & $111.3^{d}$ & -3.44 & -6.84 \\
\hline $\mathrm{Cs}^{+}$(aq) & $-264.0^{c}$ & $133.1^{d}$ & -3.08 & 0.75 \\
\hline
\end{tabular}

${ }^{a}$ Data obtained from Ref. 63; ${ }^{b}$ Data extracted from Ref. 64; ${ }^{c}$ Data extracted from Ref. 65; ${ }^{d}$ Data extracted from Ref. 62.

The change of the Gibbs free energy of the second step

$$
\Delta G_{2}=G_{T, \mathrm{MA}^{+}(\mathrm{aq})}^{\circ}+G_{T, \mathrm{I}^{-}(\mathrm{aq})}^{\circ}-G_{T, \mathrm{MA}^{+}(\mathrm{g})}^{\circ}+G_{T, \mathrm{I}^{-}(\mathrm{g})}^{\circ}
$$

amounts to $\Delta G_{2}=-5.36 \mathrm{eV}$. The strongly negative change of Gibbs free energy in the second step overcomes the Gibbs free energy gain in the first step. Combining the two-step Gibbs free energy change, we can obtain $\Delta G_{\text {diss }}=\Delta G_{1}+\Delta G_{2}=-0.36 \mathrm{eV}$. The negative $\Delta G_{\text {diss }}$ of the dissolution $\mathrm{MAPbI}_{3}$ in water at a finite concentration indicates the reaction in Eq. (7) would proceed spontaneously. The thermodynamic analysis of $\mathrm{MAPbI}_{3}$ dissolution process suggests an intrinsic water instability of $\mathrm{MAPbI}_{3}$. Although the negative $\Delta G_{\text {diss }}$ reflects a spontaneity of a reaction, it only predicts the trend of the proposed reaction. According to Arrhenius equation, the rate of a reaction is controlled by the energy barrier, i.e activation energy. A low activation energy indicates a high rate constant. The low activation energy obtained from metadynamic calculations for the $\mathrm{MAPbI}_{3}$ degradation in water demonstrates the corresponding reaction will proceed quickly. In all, for the reaction of $\mathrm{MAPbI}_{3}$ in water, the negative $\Delta G_{\text {diss }}$ renders a thermodynamic instability and the low energy barrier points to a kinetic instability.

Compared with a large amount of discussions on the fragility of $\mathrm{MAPbI}_{3}$ in water, stability 
of $\mathrm{CsPb}_{3}$ in water recently attracts attention and is also under discussion. Lin et al. 49 indicated the water invasion triggered the phase transition of $\mathrm{CsPbI}_{3}$ from a high-temperature cubic phase to a low-temperature orthorhombic phase and they found that water is adsorbed on the surface without penetrating the interior of $\mathrm{CsPbI}_{3}$. Conversely, Yuan et al. $\frac{66}{6}$ observed the $\mathrm{CsPl}_{3}$ quantum dots degraded in a chamber with a wet gas flow. They confirmed the moisture was responsible for the degradation of these $\mathrm{CsPbI}_{3}$ quantum dots. Here, we employ the two-step thermodynamic cycle to the case of $\mathrm{CsPbI}_{3}$ dissolution in water to clarify these controversies.

The first step of the proposed thermodynamic cycle of dissolution $\mathrm{CsPbI}_{3}$ in water is given as

$$
\mathrm{CsPbI}_{3}(\mathrm{~s}) \stackrel{\Delta G_{1}}{\longrightarrow} \mathrm{Cs}^{+}(\mathrm{g})+\mathrm{I}^{-}(\mathrm{g})+\mathrm{PbI}_{2}(\mathrm{~s})
$$

Related thermodynamic properties of species involved in the first step of $\mathrm{CsPbI}_{3}$ dissolution in water are listed in Table 1. We can obtain the Gibbs free energy change $\Delta G_{1}=5.26 \mathrm{eV}$ for Eq. (15). The thermodynamic cycle of $\mathrm{CsPbI}_{3}$ is shown in Fig. 4p. The second step of $\mathrm{CsPbI}_{3}$ dissolution in water involves the hydration of $\mathrm{Cs}^{+}(\mathrm{g})$ and $\mathrm{I}^{-}(\mathrm{g})$

$$
\mathrm{Cs}^{+}(\mathrm{g})+\mathrm{I}^{-}(\mathrm{g})+\mathrm{PbI}_{2}(\mathrm{~s}) \stackrel{\Delta G_{2}}{\longrightarrow} \mathrm{Cs}^{+}(\mathrm{aq})+\mathrm{I}^{-}(\mathrm{aq})+\mathrm{PbI}_{2}(\mathrm{~s})
$$

Related thermodyanmic properties are shown in Table 2. Using Gibbs free energies in Table 1 and Table 2, the change of the Gibbs free energy of the second step is estimated as $\Delta G_{2}=$ $-5.60 \mathrm{eV}$. The overall $\Delta G_{\text {diss }}=\Delta G_{1}+\Delta G_{2}=-0.34 \mathrm{eV}$ for the dissolution $\mathrm{CsPbI}_{3}$ in water

$$
\mathrm{CsPbI}_{3}(\mathrm{~s}) \stackrel{\Delta G_{\mathrm{diss}}}{\longrightarrow} \mathrm{Cs}^{+}(\mathrm{aq})+\mathrm{I}^{-}(\mathrm{aq})+\mathrm{PbI}_{2}(\mathrm{~s})
$$

The above discussion indicates that the orthorhombic $\mathrm{CsPbI}_{3}$ is also prone to decompose in water. The thermodynamic analysis of $\mathrm{CsPbI}_{3}$ dissolution in water corroborates the degradation of $\mathrm{CsPbI}_{3}$ quantum dots observed by Yuan et al. $\frac{66}{}$. The energy barrier of the 


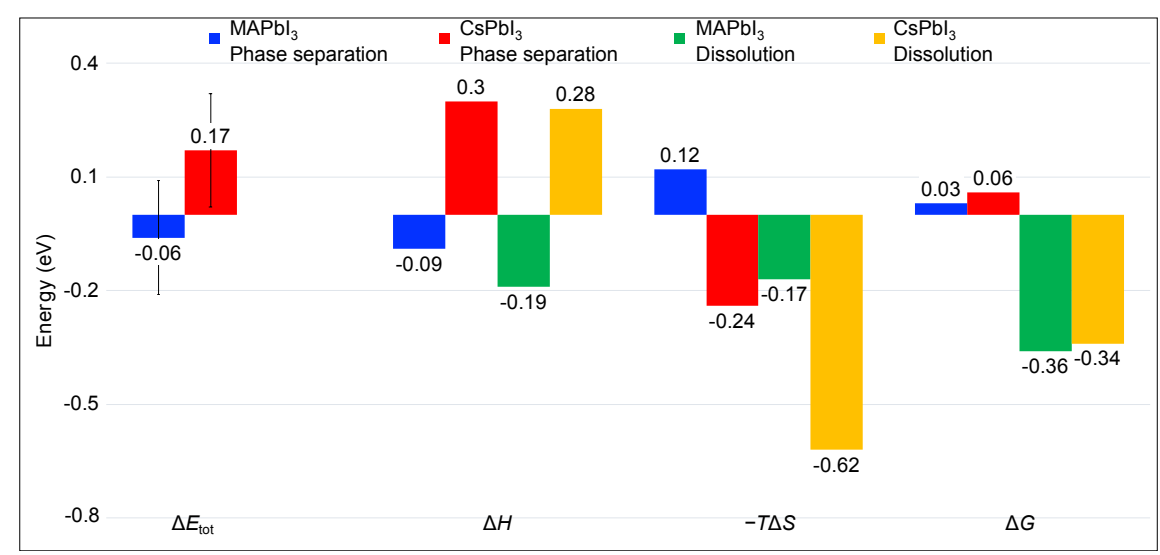

Figure 5: Thermodynamic quantities for decomposition of $\mathrm{MAPbI}_{3}$ and $\mathrm{CsPbI}_{3}$ via two alternative routes: a phase separation [Eq. (18)] or a dissolution in water [Eq. (7)]. $\Delta E_{\text {tot }}$ is the bare-DFT energy difference between products and the reactant (perovskite). The errorbars indicate a chemical uncertainty of the DFT exchange-correlation functional (see text for details). $\Delta H$ is the enthalpy change at $T=298.15 \mathrm{~K} . T \Delta S$ captures the entropy change during decomposition. $\Delta G$ is the resultant Gibbs free energy change of $\mathrm{MAPbI}_{3}$ and $\mathrm{CsPbI}_{3}$ phase separation and dissolution in water.

dissolution process determines the rate of the reaction. Compared with the very low energy barrier (about $0.18 \mathrm{eV}$ ) for the initial dissolution of $\mathrm{MAPbI}_{3}$, the relatively high energy barrier (greater than $0.5 \mathrm{eV}^{\underline{49}}$ ) for releasing $\mathrm{I}^{-}$into water from $\mathrm{CsPbI}_{3}$ surface suggests a slow process of dissolution of $\mathrm{CsPbI}_{3}$ which explains the differences in degradation rates at heterogeneous interfaces: water $/ \mathrm{MAPbI}_{3}$ vs water $/ \mathrm{CsPbI}$ observed by Lin et al. $\frac{49}{4}$.

Zhang et al. ${ }^{67}$ first used the energy differences obtained from DFT calculation to characterize the intrinsic instability of $\mathrm{MAPbI}_{3}$ and $\mathrm{CsPbI}_{3}$, considering a decomposition reaction of $\mathrm{MAPbI}_{3}$ into solid state products

$$
\mathrm{MAPbI}_{3}(\mathrm{~s}) \rightarrow \mathrm{MAI}(\mathrm{s})+\mathrm{PbI}_{2}(\mathrm{~s})
$$

In Fig. 5 we show our calculated $\Delta E_{\text {tot }}$ for $\mathrm{MAPbI}_{3}$ and $\mathrm{CsPbI}_{3}$ which match well with $\Delta E_{\text {tot }}$ from Zhang et al. $\frac{67}{}$. According to Zhang et al. $\frac{67}{}$, a positive value of $\Delta E_{\text {tot }}$ corresponds to a stable perovskite structure. They proposed the entropy term of MAI is higher than that of $\mathrm{MAPbI}_{3}$ which will further destabilize $\mathrm{MAPbI}_{3}$. However, from the measurements of entropies of MAI and $\mathrm{MAPbI}_{3}$ listed in Table 1, it clearly indicates a reverse trend. To note 
that, the entropy contribution of $\mathrm{PbI}_{2}$ cannot be neglected. The overall entropy in reaction (18) decreases and stabilizes $\mathrm{MAPbI}_{3}$ which has been indicated in Ref. 56. The entropy gains during $\mathrm{CsPbI}_{3}$ phase separation which is contrary to $\mathrm{MAPbI}_{3}$. The enthalpy change of $\mathrm{CsPbI}_{3}$ phase separation is large enough to overcome this entropy gain resulting in a positive $\Delta G$ that is slightly greater than the value of $\mathrm{MAPbI}_{3}$.

The positive $\Delta G$ values for phase separation process in Fig. 5 indicate a feasibility of synthesis of $\mathrm{MAPbI}_{3}$ and $\mathrm{CsPbI}_{3}$ compounds. However, the result should be taken with caution, since the uncertainty in reaction energies obtained with Perdew, Burke, and Ernzerhof $(\mathrm{PBE})^{27}$ exchange-correlation functional is of the order of $\pm 0.03 \mathrm{eV} /$ atom. $\frac{68}{6 i t h}$ five atoms per perovskite formula unit $\left(\mathrm{MA}^{+}\right.$is considered as one cation), the estimated error is about $\pm 0.15 \mathrm{eV}$ as shown by the errorbars in Fig. 5. When considering a water environment, the Gibbs free energy change of the decomposition reaction mentioned above decreased by $0.39 \mathrm{eV}$ and becomes strongly negative. This decrease is due to hydration of $\mathrm{MA}^{+} / \mathrm{Cs}^{+}$and $\mathrm{I}^{-}$ions. The amount of enthalpy change during the designed two-step thermodynamic cycle for $\mathrm{MAPbI}_{3}$ dissolution in water corresponding to Eq. (7) is negative (see $\Delta H$ in Fig. 5). This $\Delta H$ indicates the released energy during hydration process of $\mathrm{MA}^{+}$and $\mathrm{I}^{-}$in Eq. (12) overcomes the energy consumed in Eq. (9). It renders the dissolution $\mathrm{MAPbI}_{3}$ in water is an exothermic reaction. The entropy gain during the dissolution of $\mathrm{MAPbI}_{3}$ in water further lowers $\Delta G$ in Fig. 5, suggesting the dissolution process is thermodynamically preferable. Dissimilarly, a postive $\Delta H=0.28 \mathrm{eV}$ for $\mathrm{CsPbI}_{3}$ dissolving in water indicates the released energy during hydration process of $\mathrm{Cs}^{+}$and $\mathrm{I}^{-}$in Eq. (16) is less than the energy consumed during Eq. (15). This positive $\Delta H$ renders dissolution $\mathrm{CsPbI}_{3}$ in water belongs to an endothermic reaction. Interestingly, a large entropy enhancement of dissolution $\mathrm{CsPbI}_{3}$ in water brings down $\Delta G$ to a negative value, see in Fig. 5 . This large entropy enhancement comes from the difference of a low entropy of orthrhombic $\mathrm{CsPbI}_{3}$ and a high entropy of $\mathrm{Cs}^{+}(\mathrm{aq})$. The relative high entropy of $\mathrm{MAPbI}_{3}$ and low entropy of $\mathrm{CsPbI}_{3}$ in Table. 1 are consistent with the strong anharmonicity of $\mathrm{MAPbI}_{3}$ and weak anharmonicity of $\mathrm{CsPbI}_{3}$ 
recently disscussed in Ref. 69,70. Compared with $\mathrm{CsPbI}_{3}$, the large hydration enthalpy of $\mathrm{MA}^{+}$ion and the entropy gain together direct the negative $\Delta G_{\text {diss. }}$. In all, we can conclude that the large hydration enthalpies of $\mathrm{MA}^{+} / \mathrm{Cs}^{+}$and $\mathrm{I}^{-}$and entropy enhancement as well as the low lattice energies of halide perovskite structures are responsible for the degradation of $\mathrm{MAPbI}_{3} / \mathrm{CsPbI}_{3}$ in water.

So far, we did not discuss an ionic activity $a_{ \pm}$of $\mathrm{CH}_{3} \mathrm{NH}_{3} \mathrm{I}$ solution and its contribution $k_{B} T \ln \left(a_{ \pm}\right)$to a chemical potential term of an electrolyte when estimating $\tilde{\mu}_{T}^{\circ}(\mathrm{aq})$. The actual chemical potential of an electrolyte is expressed as

$$
\mu_{T} \approx \tilde{\mu}_{T}^{\circ}(\mathrm{aq})+k_{B} T \ln \left(a_{ \pm}\right) .
$$

The mean ionic activity $a_{ \pm}$of $\mathrm{CH}_{3} \mathrm{NH}_{3} \mathrm{I}$ solution

$$
a_{ \pm}=\left(\gamma_{ \pm} c / c^{\circ}\right)^{2}
$$

is determined by its molar concentration $c$ relative to the concentration in standard state $c^{\circ}=1 \mathrm{M}$ and the mean ionic activity coefficient $\gamma_{ \pm}$, which accounts for non-ideality of the solution. Thermodynamic data listed in Table 1 correspond to $c=1 \mathrm{M}$. Bonner $\frac{71}{71}$ and Belvèze et al. ${ }^{72}$ reported $\gamma_{ \pm}$of $\mathrm{MACl}$ is 0.58 when $c=1 \mathrm{M}$. This value is taken as an estimation of $\gamma_{ \pm}$for MAI. The additional term $k_{\mathrm{B}} T \ln \left(a_{ \pm}\right)$for MAI solution is estimated as $-0.028 \mathrm{eV}$ at $c=c^{\circ}$, which is relatively small considering $\Delta G_{\text {diss }}=-0.36 \mathrm{eV}$. In a dilute solution limit $(c \ll 1 \mathrm{M})$, the term $k_{B} T \ln \left(a_{ \pm}\right)$becomes negative and drives the water decomposition reaction of $\mathrm{MAPbI}_{3} \cdot{ }^{56}$ When decomposition proceeds to reach a finite concentration $\left(e . g ., c=c^{\circ}\right)$, the $\Delta G_{\text {diss }}$ is no longer governed by $k_{B} T \ln \left(a_{ \pm}\right)$but dominant by enthalpies and entropies of aqueous ions (see $\Delta H$ and $-T \Delta S$ of dissolution process in Fig. 5). Upon further dissolution, chemical potentials of the aqueous solution increase until $\Delta G_{\text {diss }}=0 \mathrm{eV}$, the solution is then saturated and the dissolution process ceases. 


\section{Conclusion}

The water instability of $\mathrm{MAPbI}_{3}$ is a major problem for commercialization of perovskite photovoltaics. Explanations of the underline mechanism are under debate. Here, we use $a b$ initio metadynamic method to reconstruct the free energy surface of the dissolution process of $\mathrm{MAPbI}_{3}$ in explicit water. The predictive power of metadynamics unravels the pathway of water dissolving $\mathrm{MAPbI}_{3}$ surface. One intermediate state and two transition states are identified during the initial dissolution process. The first transition state involves breaking of $\mathrm{I}-\mathrm{Pb}$ bond and formation of an intermediate state (I-MA interactions) at the surface with a low energy barrier of $0.16 \mathrm{eV}$. The second transition state corresponds to dissociation I-MA interaction and hydration of the $\mathrm{I}^{-}$ion with the energy barrier of $0.22 \mathrm{eV}$. In addition, using DFT calculations augmented with experimental data, the analysis of thermodynamics of $\mathrm{MAPbI}_{3}$ decomposition in water at a finite concentration indicates a negative Gibbs free energy change which suggests the spontaneity of water dissolution of $\mathrm{MAPbI}_{3}$. It is worthy to mention that a large hydration enthalpy of $\mathrm{MA}^{+}$and a entropy gain under aqueous condition direct the negative $\Delta G_{\text {diss }}$. Combined with the low energy barrier for ease of ions escaping from the $\mathrm{MAPbI}_{3}$ surface and spontaneous nature of dissolving in water, it can be explained why the water immediately destroys pristine $\mathrm{MAPbI}_{3}$. We also analyze how $\mathrm{CsPbI}_{3}$ react with water from the thermodynamic point of view. It is found the Gibbs free energy change of dissolution $\mathrm{CsPbI}_{3}$ in water is also a negative value similar as the value of $\mathrm{MAPbI}_{3}$, which is consistent with an experimental observation of $\mathrm{CsPbI}_{3}$ degradation in a moist environment. Compared with $\mathrm{MAPbI}_{3}$, a large entropy enhancement of $\mathrm{Cs}^{+}$

dominates the negative $\Delta G_{\text {diss }}$ for $\mathrm{CsPbI}_{3}$ dissolution in water. Our explanation provides a deeper insight of the water instability of $\mathrm{MAPbI}_{3}$ and presents a perspective on improving the instability of perovskite photovoltaics in future. 


\section{Supporting Information Available}

The following files are available free of charge.

The following files are available free of charge.

- Initial FES of $\mathrm{MA}^{+}$: The initial basin of free energy surface for $\mathrm{MA}^{+}$dissolution in water is plotted and compared with the initial basin of $\mathrm{I}^{-}$dissolution in water.

\section{Acknowledgement}

Authors are indebted to Prof. Claudine Katan, Dr. Mikaël Kepenekian and Prof. Xavier Rocquefelte from Université de Rennes 1 for advises on setting up the initial perovskite-water interface. The authors also thank Natural Sciences and Engineering Research Council of Canada under the Discovery Grant Programs RGPIN-2015-04518. All the calculations were performed using a Compute Canada infrastructure supported by the Canada Foundation for Innovation under the John R. Evans Leaders Fund program.

\section{References}

(1) Kojima, A.; Teshima, K.; Shirai, Y.; Miyasaka, T. Organometal Halide Perovskites as Visible-Light Sensitizers for Photovoltaic Cells. J. Am. Chem. Soc. 2009, 131, 60506051.

(2) Hailegnaw, B.; Kirmayer, S.; Edri, E.; Hodes, G.; Cahen, D. Rain on Methylammonium Lead Iodide Based Perovskites: Possible Environmental Effects of Perovskite Solar Cells. J. Phys. Chem. Lett. 2015, 6, 1543-1547.

(3) Li, Y.; Xu, X.; Wang, C.; Wang, C.; Xie, F.; Yang, J.; Gao, Y. Degradation by Exposure of Coevaporated $\mathrm{CH}_{3} \mathrm{NH}_{3} \mathrm{PbI}_{3}$ Thin Films. J. Phys. Chem. C 2015, 119, 23996-24002. 
(4) Ke, J. C.-R.; Walton, A. S.; Lewis, D. J.; Tedstone, A.; O’Brien, P.; Thomas, A. G.; Flavell, W. R. In situ Investigation of Degradation at Organometal Halide Perovskite Surfaces by X-ray Photoelectron Spectroscopy at Realistic Water Vapour Pressure. Chem. Commun. 2017, 53, 5231-5234.

(5) Kakekhani, A.; Katti, R. N.; Rappe, A. M. Water in Hybrid Perovskites: Bulk MAPbI Degradation via Super-Hydrous State. APL Mater. 2019, 7, 041112.

(6) Philippe, B.; Park, B.-W.; Lindblad, R.; Oscarsson, J.; Ahmadi, S.; Johansson, E. M.; Rensmo, H. Chemical and Electronic Structure Characterization of Lead Halide Perovskites and Stability Behavior under Different Exposures A Photoelectron Spectroscopy Investigation. Chem. Mater. 2015, 27, 1720-1731.

(7) Niu, G.; Li, W.; Meng, F.; Wang, L.; Dong, H.; Qiu, Y. Study on the Stability of $\mathrm{CH}_{3} \mathrm{NH}_{3} \mathrm{PbI}_{3}$ Films and the Effect of Post-Modification by Aluminum Oxide in AllSolid-State Hybrid Solar Cells. J. Mater. Chem. A 2014, 2, 705-710.

(8) Frost, J. M.; Butler, K. T.; Brivio, F.; Hendon, C. H.; Van Schilfgaarde, M.; Walsh, A. Atomistic Origins of High-Performance in Hybrid Halide Perovskite Solar Cells. Nano Lett. 2014, 14, 2584-2590.

(9) Wei, W.; Hu, Y. H. Catalytic Role of $\mathrm{H}_{2} \mathrm{O}$ in Degradation of Inorganic-Organic Perovskite $\left(\mathrm{CH}_{3} \mathrm{NH}_{3} \mathrm{PbI}_{3}\right)$ in Air. Int. J. Energy Res. 2016, 41, 1063-1069.

(10) McLeod, J. A.; Liu, L. Prospects for Mitigating Intrinsic Organic Decomposition in Methylammonium Lead Triiodide Perovskite. J. Phys. Chem. Lett. 2018, 9, 2411-2417.

(11) Yang, J.; Yuan, Z.; Liu, X.; Braun, S.; Li, Y.; Tang, J.; Gao, F.; Duan, C.; Fahlman, M.; Bao, Q. Oxygen- and Water-Induced Energetics Degradation in Organometal Halide Perovskites. ACS Appl. Mater. Interfaces 2018, 10, 16225-16230. 
(12) Kosasih, F. U.; Ducati, C. Characterising Degradation of Perovskite Solar Cells Through In-Situ and Operando Electron Microscopy. Nano Energy 2018, 47, 243-256.

(13) Aristidou, N.; Eames, C.; Islam, M. S.; Haque, S. A. Insights into the Increased Degradation Rate of $\mathrm{CH}_{3} \mathrm{NH}_{3} \mathrm{PbI}_{3}$ Solar Cells in Combined Water and $\mathrm{O}_{2}$ Environments. $J$. Mater. Chem. A 2017, 5, 25469-25475.

(14) Smecca, E.; Numata, Y.; Deretzis, I.; Pellegrino, G.; Boninelli, S.; Miyasaka, T.; Magna, A. L.; Alberti, A. Stability of Solution-Processed $\mathrm{MAPbI}_{3}$ and $\mathrm{FAPbI}_{3}$ Layers. Phys. Chem. Chem. Phys. 2016, 18, 13413-13422.

(15) Saidaminov, M. I.; Kim, J.; Jain, A.; Quintero-Bermudez, R.; Tan, H.; Long, G.; Tan, F.; Johnston, A.; Zhao, Y.; Voznyy, O. et al. Suppression of Atomic Vacancies via Incorporation of Isovalent Small Ions to Increase the Stability of Halide Perovskite Solar Cells in Ambient Air. Nat. Energy 2018, 3, 648-654.

(16) Lin, C.-C.; Lyu, Y.; Hunston, D. L.; Kim, J. H.; Wan, K.-T.; Stanley, D. L.; Gu, X. Cracking and Delamination Behaviors of Photovoltaic Backsheet after Accelerated Laboratory Weathering. Reliability of Photovoltaic Cells, Modules, Components, and Systems VIII. 2015; p 956304.

(17) Mosconi, E.; Azpiroz, J. M.; De Angelis, F. Ab Initio Molecular Dynamics Simulations of Methylammonium Lead Iodide Perovskite Degradation by Water. Chem. Mater. 2015, 27, 4885-4892.

(18) Caddeo, C.; Marongiu, D.; Meloni, S.; Filippetti, A.; Quochi, F.; Saba, M.; Mattoni, A. Hydrophilicity and Water Contact Angle on Methylammonium Lead Iodide. Adv. Mater. Interfaces 2018, 1801173.

(19) Caddeo, C.; Saba, M. I.; Meloni, S.; Filippetti, A.; Mattoni, A. Collective Molecular Mechanisms in the $\mathrm{CH}_{3} \mathrm{NH}_{3} \mathrm{PbI}_{3}$ Dissolution by Liquid Water. ACS nano 2017, 11, 9183-9190. 
(20) Park, S.; Chang, W. J.; Lee, C. W.; Park, S.; Ahn, H.-Y.; Nam, K. T. Photocatalytic Hydrogen Generation from Hydriodic Acid Using Methylammonium Lead Iodide in Dynamic Equilibrium with Aqueous Solution. Nat. Energy 2017, 2, 16185.

(21) Liu, L.-M.; Laio, A.; Michaelides, A. Initial Stages of Salt Crystal Dissolution Determined with Ab Initio Molecular Dynamics. Phys. Chem. Chem. Phys. 2011, 13, 13162-13166.

(22) Chen, J.-C.; Reischl, B.; Spijker, P.; Holmberg, N.; Laasonen, K.; Foster, A. S. Ab Initio Kinetic Monte Carlo Simulations of Dissolution at the NaCl-Water Interface. Phys. Chem. Chem. Phys. 2014, 16, 22545-22554.

(23) Laio, A.; Parrinello, M. Escaping Free-Energy Minima. Proc. Natl. Acad. Sci. U.S.A. 2002, 99, 12562-12566.

(24) Iannuzzi, M.; Laio, A.; Parrinello, M. Efficient Exploration of Reactive Potential Energy Surfaces Using Car-Parrinello Molecular Dynamics. Phys. Rev. Lett. 2003, 90, 238302.

(25) Bucko, T. Ab Initio Calculations of Free-Energy Reaction Barriers. J. Phys.: Condens. Matter 2008, 20, 064211.

(26) Kohn, W.; Sham, L. J. Self-Consistent Equations Including Exchange and Correlation Effects. Phys. Rev. 1965, 140, A1133.

(27) Perdew, J. P.; Burke, K.; Ernzerhof, M. Generalized Gradient Approximation Made Simple. Phys. Rev. Lett. 1996, 77, 3865.

(28) Wang, Y.; Gould, T.; Dobson, J. F.; Zhang, H.; Yang, H.; Yao, X.; Zhao, H. Density Functional Theory Analysis of Structural and Electronic Properties of Orthorhombic Perovskite $\mathrm{CH}_{3} \mathrm{NH}_{3} \mathrm{PbI}_{3}$. Phys. Chem. Chem. Phys. 2013, 16, 1424-1429.

(29) Zheng, C.; Rubel, O. Aziridinium Lead Iodide: A Stable, Low-Band-Gap Hybrid Halide Perovskite for Photovoltaics. J. Phys. Chem. Lett. 2018, 9, 874-880. 
(30) Li, Y.; Yang, K. High-Throughput Computational Design of Organic-Inorganic Hybrid Halide Semiconductors beyond Perovskites for Optoelectronics. Energy Environ. Sci. 2019, 12, 2233-2243.

(31) Klimeš, J.; Bowler, D. R.; Michaelides, A. Chemical Accuracy for the Van Der Waals Density Functional. J Phys. Condens. Matter 2009, 22, 022201.

(32) Grimme, S.; Antony, J.; Ehrlich, S.; Krieg, H. A Consistent and Accurate Ab Initio Parametrization of Density Functional Dispersion Correction (DFT-D) for the 94 Elements H-Pu. J. Chem. Phys. 2010, 132, 154104.

(33) Kresse, G.; Furthmüller, J. Efficient Iterative Schemes for Ab Initio Total-Energy Calculations Using A Plane-Wave Basis Set. Phys. Rev. B 1996, 54, 11169.

(34) Kresse, G.; Joubert, D. From Ultrasoft Pseudopotentials to the Projector AugmentedWave Method. Phys. Rev. B 1999, 59, 1758.

(35) Blöchl, P. Projector Augmented-Wave Method. Phys. Rev. B 1994, 50, 17953.

(36) Zheng, C.; Rubel, O. Ionization Energy as a Stability Criterion for Halide Perovskites. J. Phys. Chem. C 2017, 121, 11977-11984.

(37) Monkhorst, H. J.; Pack, J. D. Special Points for Brillouin-Zone Integrations. Phys. Rev. B 1976, 13, 5188-5192.

(38) Zheng, C.; Yu, S.; Rubel, O. Structural Dynamics in Hybrid Halide Perovskites: Bulk Rashba Splitting, Spin Texture, and Carrier Localization. Phys. Rev. Materials 2018, 2, 114604 .

(39) Whitfield, P.; Herron, N.; Guise, W.; Page, K.; Cheng, Y.; Milas, I.; Crawford, M. Structures, Phase Transitions and Tricritical Behavior of the Hybrid Perovskite Methyl Ammonium Lead Iodide. Sci. Rep. 2016, 6, 35685. 
(40) She, L.; Liu, M.; Zhong, D. Atomic Structures of $\mathrm{CH}_{3} \mathrm{NH}_{3} \mathrm{PbI}_{3}$ (001) Surfaces. ACS Nano 2015, 10, 1126-1131.

(41) Ohmann, R.; Ono, L. K.; Kim, H.-S.; Lin, H.; Lee, M. V.; Li, Y.; Park, N.-G.; Qi, Y. Real-Space Imaging of the Atomic Structure of Organic-Inorganic Perovskite. J. Am. Chem. Soc. 2015, 137, 16049-16054.

(42) Nosé, S. A Unified Formulation of the Constant Temperature Molecular Dynamics Methods. J. Chem. Phys. 1984, 81, 511-519.

(43) Hoover, W. G. Canonical Dynamics: Equilibrium Phase-Space Distributions. Phys. Rev. A 1985, 31, 1695.

(44) Ensing, B.; Laio, A.; Parrinello, M.; Klein, M. L. A Recipe for the Computation of the Free Energy Barrier and the Lowest Free Energy Path of Concerted Reactions. J. Phys. Chem. B 2005, 109, 6676-6687.

(45) Karmakar, A.; Chandra, A. Water in Hydration Shell of An Iodide Ion: Structure and Dynamics of Solute-Water Hydrogen Bonds and Vibrational Spectral Diffusion from First-Principles Simulations. J. Phys. Chem. C 2015, 119, 8561-8572.

(46) Bonomi, M.; Branduardi, D.; Bussi, G.; Camilloni, C.; Provasi, D.; Raiteri, P.; Donadio, D.; Marinelli, F.; Pietrucci, F.; Broglia, R. A. PLUMED: A Portable Plugin for Free-Energy Calculations with Molecular Dynamics. Comput. Phys. Commun. 2009, 180, 1961-1972.

(47) Momma, K.; Izumi, F. VESTA 3 for Three-Dimensional Visualization of Crystal, Volumetric and Morphology Data. J. Appl. Crystallogr. 2011, 44, 1272-1276.

(48) Markovich, G.; Giniger, R.; Levin, M.; Cheshnovsky, O. Photoelectron Spectroscopy of Iodine Anion Solvated in Water Clusters. J. Chem. Phys. 1991, 95, 9416-9419. 
(49) Lin, J.; Lai, M.; Dou, L.; Kley, C. S.; Chen, H.; Peng, F.; Sun, J.; Lu, D.; Hawks, S. A.; Xie, C. Thermochromic Halide Perovskite Solar Cells. Nat. Mater. 2018, 17, 261-267.

(50) Ohtaki, H.; Fukushima, N.; Hayakawa, E.; Okada, I. Dissolution Process of Sodium Chloride Crystal in Water. Pure Appl. Chem. 1988, 60, 1321-1324.

(51) Yang, Y.; Meng, S.; Xu, L. F.; Wang, E. G.; Gao, S. Dissolution Dynamics of NaCl Nanocrystal in Liquid Water. Phys. Rev. E 2005, 72, 012602.

(52) Fedotova, M.; Kruchinin, S. Hydration of Methylamine and Methylammonium Ion: Structural and Thermodynamic Properties from the Data of the Integral Equation Method in the RISM Approximation. Russ. Chem. Bull. 2012, 61, 240-247.

(53) Delugas, P.; Filippetti, A.; Mattoni, A. Methylammonium Fragmentation in Amines as Source of Localized Trap Levels and the Healing Role of $\mathrm{Cl}$ in Hybrid Lead-Iodide Perovskites. Phys. Rev. B 2015, 92, 045301.

(54) Fan, Z.; Xiao, H.; Wang, Y.; Zhao, Z.; Lin, Z.; Cheng, H.-C.; Lee, S.-J.; Wang, G.; Feng, Z.; Goddard III, W. A. Layer-by-Layer Degradation of Methylammonium Lead Tri-iodide Perovskite Microplates. Joule 2017, 1, 548-562.

(55) Sholl, D.; Steckel, J. A. Density Functional Theory: a Practical Introduction; John Wiley \& Sons: Hoboken, New Jersey, 2011.

(56) Tenuta, E.; Zheng, C.; Rubel, O. Thermodynamic Origin of Instability in Hybrid Halide Perovskites. Sci. Rep. 2016, 6, 37654.

(57) Kye, Y.-H.; Yu, C.-J.; Jong, U.-G.; Ri, K.-C.; Kim, J.-S.; Choe, S.-H.; Hong, S.-N.; Li, S.; Wilson, J. N.; Walsh, A. Vacancy-Driven Stabilization of the Cubic Perovskite Polymorph of $\mathrm{CsPbI}_{3}$. J. Phys. Chem. C 2019, 123, 9735-9744.

(58) Onoda-Yamamuro, N.; Matsuo, T.; Suga, H. Calorimetric and IR Spectroscopic Studies 
of Phase Transitions in Methylammonium Trihalogenoplumbates (II). J. Phys. Chem. Solids 1990, 51, 1383-1395.

(59) Ong, S. P.; Cholia, S.; Jain, A.; Brafman, M.; Gunter, D.; Ceder, G.; Persson, K. A. The Materials Application Programming Interface (API): a Simple, Flexible and Efficient API for Materials Data Based on REpresentational State Transfer (REST) Principles. Comput. Mater. Sci. 2015, 97, 209-215.

(60) Hinuma, Y.; Pizzi, G.; Kumagai, Y.; Oba, F.; Tanaka, I. Band Structure Diagram Paths Based on Crystallography. Comput. Mater. Sci. 2017, 128, 140-184.

(61) Yamamuro, O.; Oguni, M.; Matsuo, T.; Suga, H. Calorimetric and Dilatometric Studies on the Phase Transitions of Crystalline $\mathrm{CH}_{3} \mathrm{NH}_{3} \mathrm{I}$. J. Chem. Thermodyn. 1986, 18, 939954.

(62) Rumble, J. CRC Handbook of Chemistry and Physics; CRC press: Boca Raton, Florida, 2017.

(63) Housecroft, C. E.; Jenkins, H. D. B. Absolute Ion Hydration Enthalpies and the Role of Volume within Hydration Thermodynamics. RSC Adv. 2017, 7, 27881-27894.

(64) Marcus, Y.; Loewenschuss, A. Chapter 4. Standard Entropies of Hydration of Ions. Annu. Rep. Prog. Chem., Sect. C: Phys. Chem. 1984, 81, 81-135.

(65) Smith, D. W. Ionic Hydration Enthalpies. J. Chem. Educ. 1977, 54, 540-542.

(66) Yuan, G.; Ritchie, C.; Ritter, M.; Murphy, S.; Gómez, D. E.; Mulvaney, P. The Degradation and Blinking of Single $\mathrm{CsPbI}_{3}$ Perovskite Quantum Dots. J. Phys. Chem. C 2017, 122, 13407-13415.

(67) Zhang, Y.; Chen, S.; Xu, P.; Xiang, H.; Gong, X.; Walsh, A.; Wei, S. Intrinsic Instability of the Hybrid Halide Perovskite Semiconductor $\mathrm{CH}_{3} \mathrm{NH}_{3} \mathrm{PbI}_{3}$. Chin. Phys. Lett. 2018, 35,036104 . 
(68) Hautier, G.; Ong, S. P.; Jain, A.; Moore, C. J.; Ceder, G. Accuracy of Density Functional Theory in Predicting Formation Energies of Ternary Oxides from Binary Oxides and Its Implication on Phase Stability. Phys. Rev. B 2012, 85, 155208.

(69) Zhu, T.; Ertekin, E. Mixed Phononic and Non-Phononic Transport in Hybrid Lead Halide Perovskites: Glass-Crystal Duality, Dynamical Disorder, and Anharmonicity. Energy Environ. Sci. 2019, 12, 216-229.

(70) Marronnier, A.; Roma, G.; Boyer-Richard, S.; Pedesseau, L.; Jancu, J.-M.; Bonnassieux, Y.; Katan, C.; Stoumpos, C. C.; Kanatzidis, M. G.; Even, J. Anharmonicity and Disorder in the Black Phases of Cesium Lead Iodide Used for Stable Inorganic Perovskite Solar Cells. ACS nano 2018, 12, 3477-3486.

(71) Bonner, O. D. Osmotic and Activity Coefficients of Methyl-Substituted Ammonium Chlorides. J. Chem. Soc. Faraday Trans. 1981, 77, 2515-2518.

(72) Belvèze, L. S.; Brennecke, J. F.; Stadtherr, M. A. Modeling of Activity Coefficients of Aqueous Solutions of Quaternary Ammonium Salts with the Electrolyte-NRTL Equation. Ind. Eng. Chem. Res 2004, 43, 815-825. 


\section{Graphical TOC Entry}

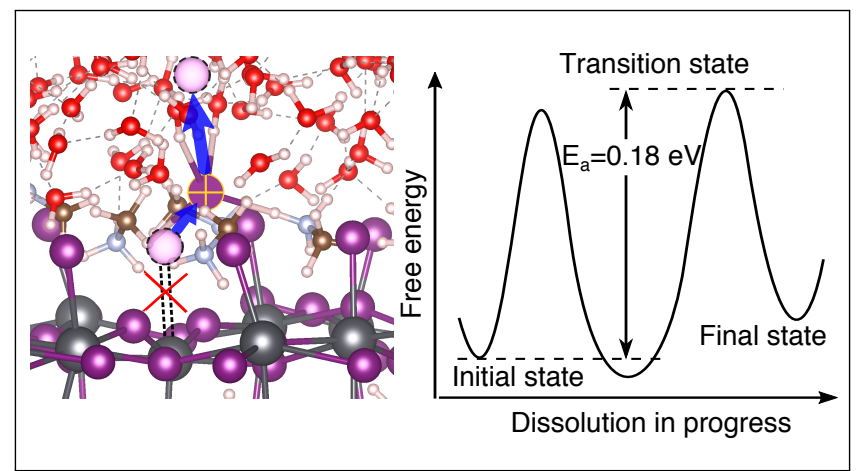

